\title{
Metabolite transporter and its impact on metabolic engineering approaches
}

UNSOLICITED REVIEWE

\author{
Corresponding author: \\ Dr. Mostafa G. FadL , PhD. of Microbiology \\ Nuclear Material Authority, \\ Mostafa_gomaa338@yahoo.com
}

\begin{abstract}
:
Metabolite transport across cellular membranes is a key feature of living organisms. Specialized proteins or protein complexes mediate such transport processes and are accessible to genetic engineering approaches. This special issue will reflect on the different transport between cellular compartments. It will provide an overview about different transporter classes and metabolic engineering approaches in different organisms ranging from Bacteria to Eukarya. The transport mechanisms energetics of these compounds were thought little of, and most consideration was given to the designing of metabolic pathways. Gram-negative microbes discharge a wide scope of proteins whose capacities incorporate biogenesis of organelles, for example, pilli and flagella, supplement securing, destructiveness, and efflux of medications and different poisons. Six particular emission frameworks have been appeared to intervene protein send out through the inward and external layers of Gram-negative microbes. These pathways are exceptionally monitored all through the Gram-negative bacterial species. In Gram-positive microscopic organisms, discharged proteins are usually moved across the single layer by the Sec pathway or the two-arginine (Tat) pathway. Recently efforts focused on transporter and metabolite engineering, visualize the improvement and enhancement of microbial cell
\end{abstract}


to pass such metabolites these metabolite carrier proteins are inserted into the inner membrane of mitochondria across the inner membrane, specialized carrier proteins are necessary that mediate the transport into and out of the mitochondrial matrix. Two main strategies for metabolic engineering in plants: the introduction of genes encoding new enzymes and the use of TFs controlling specialized metabolic pathways

Key words: Gram-negative bacterial, Metabolite transporter, pathways, metabolic engineering approaches

INTRODUCTION:

1- ATP-restricting tape $(\mathrm{ABC})$ frameworks are generally circulated among living beings and capacity in a wide range of parts of bacterial physiology. $\mathrm{ABC}$ carriers are most popular for their function in the import of basic supplements and the fare of poisonous atoms, however they can likewise intercede the vehicle of numerous other physiological substrates. In a traditional vehicle response, two profoundly monitored ATP-restricting spaces or subunits couple the official/hydrolysis of ATP to the movement of specific substrates across the film, through collaborations with layer spreading over areas of the carrier. Minor departure from this fundamental topic include solvent ABC ATP-restricting proteins that couple ATP hydrolysis to non-transport measures, for example, DNA fix and quality articulation guideline. Experiences into the structure, capacity, and component of activity of bacterial $\mathrm{ABC}$ proteins are accounted for, in light 
of phylogenetic correlations just as exemplary biochemical and hereditary methodologies. These advances are likewise significant for explaining the component of activity of eukaryotic $\mathrm{ABC}$ proteins, on the grounds that practical deformities in a large number of them are liable for extreme human acquired illnesses. (2) The external layer (OM) of most Gram-negative microscopic organisms contains lipopolysaccharide (LPS) in the external pamphlet. LPS, or endotoxin, is a particle of significant natural exercises. In the host, LPS inspires a powerful safe reaction, while in the bacterium, it assumes a pivotal part by building up a boundary to restrict section of hydrophobic atoms. Before LPS is gathered at the OM, it should be orchestrated at the inward layer (IM) and shipped across the fluid periplasmic compartment. Much is thought about the biosynthesis of LPS at the same time, as of not long ago, little was thought about its vehicle and gathering. the disclosure of two fundamental Escherichia coli IM proteins of obscure capacity, YjgP and YjgQ, which are needed for the vehicle of LPS to the cell surface. We recommend that these two proteins, which we have renamed $\mathrm{LptF}$ and $\mathrm{LptG}$, individually, are the missing transmembrane parts of the $\mathrm{ABC}$ carrier that, along with LptB, capacities to separate LPS from the IM on the way to the OM. (3)

Microcin $\mathrm{C}(\mathrm{McC})$, a peptide-nucleotide anti-infection, targets aspartyltRNA synthetase. By examining an arbitrary transposon library, we recognized Escherichia coli freaks impervious to McC. Transposon inclusions were limited to a solitary locus, yejABEF, which encodes segments of a putative internal film $\mathrm{ABC}$ carrier. Investigation of siteexplicit freaks set up that every one of the four segments of the carrier are needed for McC affectability. Since aspartyl-tRNA synthetase in yej freak separates was completely delicate to $\mathrm{McC}$, we infer that yej transformations meddle with $\mathrm{McC}$ take-up and that YejABEF is the lone inward layer carrier answerable for McC take-up in E. coli. Different substrates of YejABEF 
stay to be distinguished. (4) Periplasmic layer combination proteins (MFPs) are basic segments of the sort I protein discharge frameworks and medication efflux siphons in Gram-negative microbes. Past examinations recommended that MFPs interface the internal and external film segments of the vehicle frameworks and by this implies co-ordinate the exchange of substrates across the two layers. In this investigation, we cleansed and reconstituted the macrolide carrier MacAB from Escherichia coli. Here, MacA is a periplasmic MFP and MacB is an ABC-type carrier. Like other MFP-subordinate carriers from E. coli, the in vivo capacity of MacAB requires the external layer channel TolC. The purged MacB showed a basal ATPase movement in cleanser micelles. This movement adjusted to Michaelis-Menten energy yet was inert to substrates or extra proteins. Upon reconstitution into proteoliposomes, the ATPase movement of MacB was carefully subject to MacA. The synergist proficiency of MacAB ATPase was more than 45-crease higher than the action of MacB alone. Both the Nand C-terminal locales of MacA were fundamental for this movement. MacA animated MacB ATPase just in phospholipid bilayers and didn't require the presence of macrolides. Our outcomes propose that MacA is a practical subunit of the MacB carrier. (5)

Evidence indicates that MacAB, an ATP-restricting tape carrier of MacB and its frill protein, MacA, takes an interest in the movement of STII from the periplasm to the outside. Since it has been accounted for that MacAB helps out TolC, we suggest that the MacAB-TolC framework catches the periplasmic STII particles and fares the poison atoms to the outside.

The MacB ABC carrier shapes a three sided efflux siphon with the MacA connector protein and TolC external film leave pipe to oust anti-toxins and fare destructiveness factors from Gram-negative microbes. Here, we survey ongoing primary and useful information on MacB and its homologs. In the 
MacAB-TolC three sided siphon, mechanotransmission drives efflux of antiinfection agents and fare of a protein poison from the periplasmic space by means of the TolC leave channel. Homologous three sided frameworks from pathogenic microscopic organisms correspondingly send out protein-like flagging particles, destructiveness factors and siderophores. What's more, numerous MacB-like ABC carriers don't frame three sided siphons, yet rather work in assorted cell measures including anti-microbial detecting, cell division and lipoprotein dealing. (6) The heat-stable enterotoxin (ST) delivered by enterotoxigenic Escherichia coli is an extracellular peptide poison that summons watery looseness of the bowels in the host. Two sorts of STs, STI and STII, have been found. Both STs are integrated as antecedent proteins and are then changed over to the dynamic structures with intramolecular disulfide securities in the wake of being delivered into the periplasm. The dynamic STs are at long last moved across the external film through a passage made by TolC. In any case, it is indistinct how the dynamic STs framed in the periplasm are directed to the TolC channel. A few carriers in the inward layer and their periplasmic extra proteins are known to consolidate with TolC and structure a three sided transport framework. We consequently anticipate that such carriers should likewise go about as a cooperate with TolC to trade STs from the periplasm to the outside. utilizing E. coli BL21(DE3) freaks in which different carrier qualities (acrAB, acrEF, emrAB, emrKY, mdtEF, macAB, and yojHI) had been taken out and investigated the discharge of STs in those strains. The outcomes uncovered that the extracellular emission of STII was generally diminished in the macAB freak and the poison particles were collected in the periplasm, despite the fact that the discharge of STI was not influenced in any freak. The periplasmic stagnation of STII in the macAB freak was reestablished by the presentation of $\mathrm{pACYC} 184$, containing the macAB quality, into the cell. 
2-Phosphotransferase system (PTS)

The Bacillus subtilis protein Crh displays solid similitude to HPr, a phosphocarrier protein of the phosphoenolpyruvate:sugar phosphotransferase framework (PTS). HPr phosphorylated at His-15 can move its phosphoryl gathering to a few EIIAs of the PTS for sugar transport and phosphorylation. Likewise, it phosphorylates and actuates transcriptional controllers containing PTS guideline spaces (PRDs). In Gram-positive microscopic organisms, it likewise controls the compound glycerol kinase. Since in Crh the dynamic site His-15 of HPr is supplanted with a glutamine, Crh couldn't complete the synergist and administrative capacities intervened by P roughly His-HPr. Notwithstanding, when Gln-15 of Crh was supplanted with a histidine, Crh picked up the greater part of the reactant and administrative capacities applied by HPr. To permit CrhQ15H to effectively phosphorylate and actuate the PRD-containing antiterminator LicT, which controls the declaration of the bgIS quality and the bgIPH operon, it was adequate to communicate the crhQ15H allele leveled out of the spac advertiser in monocopy. On the other hand, to phosphorylate and actuate glycerol kinase and to permit a ptsH cancellation strain (without HPr) to gradually develop on the non-PTS substrate glycerol and to productively use the PTS sugars glucose and mannitol, the crhQ15H allele must be communicated from a multicopy plasmid.(8) In Bacillus subtilis and close family members, worldwide guideline of carbon catabolite control happens on the official of the complex of CcpA (catabolite control protein A) and P-Ser-HPr (seryl-phosphorylated type of HPr) to the catabolite responsive components (cre) of the objective operons, the constituent qualities of which are generally assessed to number 300. major extracellular results of B. subtilis developed on glucose. It additionally triggers the 
declaration of an anabolic operon (ilv-leu) associated with the biosynthesis of stretched chain amino acids, which consequently prompts cell proliferation. Then again, this complex quells numerous qualities and operons, which incorporate a passage quality for the TCA cycle (citZ), a few carrier qualities for TCA cycle-intermediates, some breath qualities, and numerous catabolic and anabolic qualities engaged with carbon, nitrogen, and phosphate digestion, just as for certain extracellular proteins and optional metabolites. Another CcpA-free catabolite restraint framework includes P-His-HPr (histidyl-phosphorylated type of HPr). P-His-HPr phosphorylates and enacts glycerol kinase, whose item is important for antitermination of the glycerol use operon through GlpP, the antiterminators (LicT and SacT, Y) of a few operons for the use of less-favored PTS-sugars, and some transcriptional activators, for example, LevR for the levan use operon. This phosphorylation is diminished because of the diminished degree of P-His-HPr during dynamic vehicle of a favored PTS-starch, for example, glucose, coming about in catabolite constraint of the objective operons.Thus CcpA-needy and autonomous organizations for carbon digestion assume a significant part in the arrange guideline of catabolism and anabolism to guarantee ideal cell proliferation in the presence and the nonappearance of a favored PTS-sugar.(9) The histidine-containing protein (HPr) is the energy coupling protein of the phosphoenolpyruvatesubordinate carbohydrate:phosphotransferase framework (PTS), which catalyzes the vehicle of starches in microbes. In Bacillus subtilis and close family members, worldwide guideline of carbon catabolite control happens on the authoritative of the complex of CcpA (catabolite control protein A) and P-Ser-HPr (seryl-phosphorylated type of HPr) to the catabolite responsive components (cre) of the objective operons, the constituent qualities of which are generally assessed to number 300 . The complex of CcpA and P-Ser-HPr triggers the declaration of a few qualities associated with the arrangement of acetic acid derivation and acetoin, major 
extracellular results of B. subtilis developed on glucose. It likewise triggers the declaration of an anabolic operon (ilv-leu) associated with the biosynthesis of fanned chain amino acids, which accordingly prompts cell engendering. Then again, this complex subdues numerous qualities and operons, which incorporate a passageway quality for the TCA cycle (citZ), a few carrier qualities for TCA cycle-intermediates, some breath qualities, and numerous catabolic and anabolic qualities associated with carbon, nitrogen, and phosphate digestion, just as for certain extracellular chemicals and auxiliary metabolites. Moreover, these microbes have CcpA-autonomous catabolite guideline frameworks, every one of which includes a transcriptional repressor of $\mathrm{CggR}$ or $\mathrm{CcpN}$. $\mathrm{CggR}$ and $\mathrm{CcpN}$ are derepressed under glycolytic and gluconeogenic development conditions, and improve glycolysis and gluconeogenesis separately. Another CcpA-free catabolite suppression framework includes P-His-HPr (histidyl-phosphorylated type of HPr). P-His-HPr phosphorylates and initiates glycerol kinase, whose item is vital for antitermination of the glycerol use operon through GlpP, the antiterminators (LicT and SacT, Y) of a few operons for the use of lessfavored PTS-sugars, and some transcriptional activators, for example, LevR for the levan use operon. This phosphorylation is diminished because of the diminished degree of P-His-HPr during dynamic vehicle of a favored PTSstarch, for example, glucose, coming about in catabolite restraint of the objective operons. Thus CcpA-needy and free organizations for carbon digestion assume a significant part in the arrange guideline of catabolism and anabolism to guarantee ideal cell engendering in the presence and the nonattendance of a favored PTS-sugar (6) combination of MurP was built, disengaged from layers, and recognized as a polypeptide. Close homologs of MurP were distinguished in the genome of a few microbes, and we accept that these life forms may likewise have the option to use MurNAc.(10) The phosphoenolpyruvate-subordinate phosphotransferase framework (PTS) is the fundamental sugar take-up framework in Bacillus subtilis A run of the 
mill PTS comprises of two general proteins, compound I (EI) and a histidine-containing protein (HPr), just as a particular starch carrier (or chemical II [EII]), all of which move the phosphoryl bunch from phosphoenolpyruvate to the moved carb. The particular PTS carriers are framed by multidomain proteins or single-area subunits. These areas are space C (EIIC), the transmembrane channel for the sugar transport; space B (EIIB), the layer headed space liable for phosphorylation of the starch; and space A (EIIA), the arbiter among $\operatorname{HPr}(\mathrm{H} 15 \sim \mathrm{P})$ and EIIB. There are 16 PTS carriers. Cancellation of the single-EIIA-containing carriers demonstrated that there is cross talk between the noncognate EIIA and EIIB spaces in PTS. By cancellation of all EIIA-containing proteins, strain KM455 ( $\triangle$ EIIA) was developed, and the EIIA-containing proteins were exclusively brought into the strain. Along these lines, the PTS carriers of the glucose family, specifically, PtsG, GamP, and PtsA (otherwise called YpqE). Development of TkmA-EIIA combination proteins affirmed the plausible connection between the EIIAs of the glucose group of PTS carriers and the EIIAinadequate PTS carriers. In like manner, we have indicated that SacX is predominantly phosphorylated by PtsA and GamP. PtsG and GmuA were additionally ready to phosphorylate $\mathrm{SacX}$, The phosphoenolpyruvatesubordinate phosphotransferase framework (PTS) not exclusively is a starch take-up framework in B. subtilis yet in addition assumes a significant function in detecting the supplement vacillation in the medium. This detecting framework empowers the cells to react to these variances appropriately. The PTS carriers have a significant part in this detecting framework since they are starch explicit. (11)

In excess of 20 starches might be shipped into the bacterial cell by the phosphoenopyruvate:carbohydrate phosphotransferase framework (PTS) that is broadly spread among microbes. The PTS comprises of two cytoplasmic energy-coupling proteins (Enzyme I and HPr) and a scope of 
sugar explicit Enzymes II, which catalyze accompanying starch movement and phosphorylation. The phosphorylation status of PTS parts mirrors the accessibility of starches and the energy states of the cell. In numerous microbes, PTS and the related proteins convert this data to signals, which transduced through various systems lead to wonders of catabolite suppression, inducer control or chemotaxis. These highlights of PTS furnish microorganisms with an incorporated framework, which guarantees ideal use of sugars in complex conditions. Besides, a few microbes advanced equal frameworks that serve an administrative capacities, yet obviously don't catalyze the sugar transport. (12) Various gram-negative and grampositive microbes take up sugars through the phosphoenolpyruvate (PEP):carbohydrate phosphotransferase framework (PTS). This framework ships and phosphorylates sugars to the detriment of PEP. The proteins II are needed for the vehicle of the starches across the layer and the exchange of the phospho bunch from phospho-HPr to the sugars. Biochemical, underlying, and atomic hereditary investigations have demonstrated that the different proteins II have a similar fundamental structure. Every protein II comprises of areas for explicit capacities, e.g., official of the sugar or phosphorylation. Every chemical II complex can comprise of one to four distinct polypeptides. The proteins II can be put into in any event four classes based on succession similitude. The hereditary qualities of the PTS is unpredictable, and the statement of PTS proteins is complicatedly controlled due to the focal parts of these proteins in supplement procurement. Notwithstanding traditional acceptance suppression instruments including repressor and activator proteins, different sorts of guideline, for example, antitermination, have been seen in some PTSs. Aside from their function in sugar transport, PTS proteins are associated with chemotaxis toward PTS starches. Besides, the IIAGlc protein, part of the glucose-explicit PTS, is a focal administrative protein which in its nonphosphorylated structure can dilemma to and restrain a few non-PTS 
take-up frameworks and in this manner forestall section of inducers. In its phosphorylated structure, P-IIAGlc is engaged with the initiation of adenylate cyclase and in this manner in the guideline of quality articulation. By detecting the presence of PTS starches in the medium and changing the phosphorylation province of IIAGlc, cells can adjust rapidly to changing conditions in the climate. In gram-positive microbes, it has been shown that HPr can be phosphorylated by ATP on a serine buildup and this change may play out an administrative capacity.(13)

\section{3- Bacterial secretion system}

The utilization of discharge frameworks by microorganisms to undercut have capacities. Microscopic organisms have advanced various frameworks to collaborate with and defeat their eukaryotic host and different prokaryotes. Emission frameworks are needed for the arrival of a few effectors through the bacterial membrane(s) into the extracellular space or straightforwardly into the cytoplasm of the host. (4)

Gram-negative bacteria secrete a wide range of proteins whose functions include biogenesis of organelles, such as pilli and flagella, nutrient acquisition, virulence, and efflux of drugs and other toxins. Six distinct secretion systems have been shown to mediate protein export through the inner and outer membranes while In Gram-positive bacteria, secreted proteins are commonly translocated across the single membrane by the $\mathrm{Sec}$ pathway or the two-arginine (Tat) pathway.

A wide assortment of Gram-negative bacterial microorganisms utilize a 'type III' protein emission framework to convey bacterial harmfulness factors into have cells. Late outcomes recommend that Gram-positive microbes may utilize comparative techniques to convey destructiveness 
factors into have cells. (14)The bacterial type III emission pathway conveys effector proteins into eukaryotic cells. Investigation of the sort III framework and flagellar send out qualities in the commit parasites of the family Chlamydiales proposes that the type III framework emerged from the flagellar trade framework in chlamydiae or related microbes.(15) Gramnegative microbes use type III emission (TTS) frameworks to move proteins into the extracellular climate or straightforwardly into eukaryotic cells. These complex secretory frameworks are gathered from more than 20 distinctive primary proteins, including 10 that have partners in the flagellar send out pathway. Emission substrates are coordinated to the TTS apparatus by means of mRNA as well as amino corrosive discharge signals. TTS chaperones tie to choose emission substrates and aid the fare cycle. Late advancement in the comprehension of TTS is inspected. (16) Type IV carriers are delivered by a few bacterial microbes, for example, Agrobacterium tumefaciens, These carriers are basic for the pathogenic cycle in that they trade significant harmfulness factors across the layers of the microscopic organisms. Despite the fact that the destructiveness factors that are sent out by these carriers can be either nucleic corrosive or protein, the overall system of transport seems, by all accounts, to be comparable for individuals from this family. (17) The bacterial type IV secretion systems (T4SSs) are a practically assorted superfamily of emission frameworks found in numerous types of microorganisms. Altogether, the T4SSs move DNA and monomeric and multimeric protein substrates to bacterial and eukaryotic cell types. T4SSs are made out of two enormous subfamilies, the formation machines and the effector translocators that send their cargoes through foundation of direct contributor target cell contacts, and a third little subfamily equipped for bringing in or trading substrates from or to the milieu. This survey sums up late unthinking and underlying discoveries that are revealing new insight into how T4SSs have developed such utilitarian variety. Movement signals are currently known to be found $\mathrm{C}$ terminally or 
implanted inside in underlying folds; these signs in blend with substraterelated connector proteins intervene the docking of explicit substrate collections to related VirD4-like receptors. For the Legionella pneumophila Dot/Icm framework, ongoing work has clarified the underlying reason for connector subordinate substrate stacking onto the VirD4-like DotL receptor. Advances in meaning of T4SS machine structures currently consider definite examinations of nanomachines firmly identified with the Agrobacterium tumefaciens VirB/VirD4 T4SS with those all the more indirectly related, e.g., the Dot/Icm and Helicobacter pylori Cag T4SSs. At last, it is progressively clear that T4SSs have advanced an assortment of components reliant on elaboration of conjugative pili, layer cylinders, or surface adhesins to build up gainful contacts with target cells. T4SSs subsequently have developed outrageous practical variety through a plenty of transformations affecting substrate determination, machine design, and target cell official.(18) The as of late sequenced genome of the bacterial plant microorganism Xanthomonas axonopodis pv. citri contains two virB quality groups, one on the chromosome and one on a $64-\mathrm{kb}$ plasmid, every one of which codes for a formerly uncharacterized type IV emission framework (T4SS). Announced outcomes uncovered cooperations between referred to T4SS segments just as already uncharacterized communications including theoretical proteins coded by open perusing outlines in the two X. axonopodis pv. citri virB loci. Results demonstrate that the two loci may code for beforehand unidentified VirB7 proteins, which we show connect with either VirB6 or VirB9 or with a theoretical protein coded by a similar locus. Moreover, a bunch of beforehand uncharacterized Xanthomonas proteins have been found to cooperate with VirD4, whose quality is neighboring the chromosomal virB locus. The quality for one individual from this family is found inside the chromosomal virB locus. All these uncharacterized proteins have a saved 120-amino-corrosive area in their C ends and may speak to a group of cofactors or substrates of the 
Xanthomonas T4SS.(19) A couple of bacterial microorganisms use arrangement machines to convey effector particles during infection. Such structures are people from the sort IV or 'changed development' emanation family. The prototypical sort IV structure is the Agrobacterium tumefaciens T-DNA move machine, which passes on oncogenic nucleoprotein particles to plant cells. Various organisms, including Bordetella pertussis, Legionella pneumophila, Brucellaspp. likewise, Helicobacter pylori, use type IV machines to exchange effector proteins to the extracellular milieu or the mammalian cell cytosol. (20)

Bacterial formation frameworks are exceptionally unbridled macromolecular exchange frameworks that sway human wellbeing fundamentally. In clinical settings, formation is incredibly dangerous, prompting the fast scattering of anti-toxin opposition qualities and other harmfulness attributes among bacterial populaces. Ongoing work has demonstrated that few microorganisms of plants and warm blooded creatures - Agrobacterium tumefaciens, Bordetella pertussis, Helicobacter pylori and Legionella pneumophila - have developed emission pathways tribally identified with formation frameworks to convey effector particles to eukaryotic objective cells. Every one of these frameworks trades unmistakable DNA or protein substrates to influence a bunch of changes in host cell physiology during contamination. Aggregately, discharge pathways tribally identified with bacterial formation frameworks are presently alluded to as the sort IV emission family. The rundown of putative kind IV relatives is expanding quickly, recommending that macromolecular exchange by these frameworks is an inescapable wonder in nature. (21) Protein send out by Gram-negative microscopic organisms requires committed hardware to take into consideration the section of hydrolytic catalysts and poisons through the cell envelope. The Type II fare hardware has various unmistakable qualities, which incorporate its part as an augmentation of 
Sec-subordinate emission, its capacity to perceive and trade completely collapsed substrates effectively and, maybe most essentially, the connection between a subset of its quality items with the Type IV pilus-biogenesis device. A significant inquiry is whether we can extrapolate our insight, but restricted, of Type IV pilus biogenesis to comprehend the structure and capacity of the Type II fare device. (22) Genome sequencing of bacterial microbes keeps on distinguishing extra species that contain DNA profoundly homologous to the sort II discharge qualities, recommending that this pathway is broadly disseminated inside the proteobacterial family. This survey talks about the recognizable proof of extra kind II emission qualities and the extension of the sort II discharge quality family. The guideline of type II discharge and its part in infection are additionally tended to. At long last, the finding that a segment of the sort II discharge pathway in Vibrio cholerae can likewise uphold bacteriophage expulsion is talked about. (23) The sort II emission pathway or the primary terminal part of the overall discharge pathway, as it has additionally been alluded to, is generally appropriated among Proteobacteria, in which it is liable for the extracellular emission of poisons and hydrolytic catalysts, a significant number of which add to pathogenesis in the two plants and creatures. Emission through this pathway varies from most other film transport frameworks, in that its substrates comprise of collapsed proteins. The sort II discharge device is made out of at any rate 12 diverse quality items that are thought to shape a multiprotein complex, which ranges the periplasmic compartment and is explicitly needed for movement of the emitted proteins across the external layer. This pathway imparts numerous highlights to the sort IV pilus biogenesis framework, including the capacity to collect a pilus-like structure. This audit examines late discoveries on the association of the discharge mechanical assembly and the function of its different parts in emission. Various models for pilus-intervened discharge through the gated pore in the external layer are likewise introduced, similar to the potential 
properties that decide if a protein is perceived and emitted by the sort II pathway. (24)

Bacterial sort II discharge frameworks (T2SSs) move destructiveness elements, poisons and catalysts across the cell external film. Here we utilize negative stain and cryo-electron microscopy to uncover the center engineering of a collected T2SS from the microorganism Klebsiella pneumoniae. We show that 7 proteins structure a $\sim 2.4$ MDa complex that traverses the cell envelope. The external film complex incorporates the secretin PulD, with all areas displayed, and the pilotin PulS. Evenness jumble between the external film complex and gathering stage is overwhelmed by PulC linkers traversing the periplasm, with PulC HR areas restricting freely at the secretin base. Our outcomes show that the T2SS has a profoundly unique measured engineering, with suggestion for pseudo-pilus get together and substrate stacking.(25) In gram-negative microscopic organisms, type I discharge is completed by a translocator comprised of three proteins that length the cell envelope. One of these proteins is a particular external layer protein (OMP) and the other two are cytoplasmic film proteins: an ATP-restricting tape (ABC) and the supposed layer combination or connector protein (MFP). Type I discharge is secautonomous and sidesteps the periplasm. This far reaching pathway permits the discharge of proteins of different sizes and capacities through a Cterminal uncleaved emission signal. This $\mathrm{C}$-terminal emission signal explicitly perceives the $\mathrm{ABC}$ protein, setting off the gathering of the useful trans-envelope complex. This report will principally arrangement will late information concerning the structure and gathering of the discharge mind boggling just as the impacts and part of substrate collapsing on emission by this pathway. (26) Numerous gram-negative microscopic organisms produce meager protein fibers, named pili, which stretch out past the limits of the external film. The significance of these pili is represented by the way that 
profoundly intricate, multi-protein pilus-gathering machines have advanced, not once, yet a few times. Their numerous capacities incorporate motility, bond, emission, and DNA move, all of which can add to the destructiveness of bacterial microbes or to the spread of harmfulness factors by even quality exchange. (27)

Gram-negative microscopic organisms utilize the sort II emission framework to ship countless discharged proteins from the periplasmic space into the extracellular climate. A considerable lot of the discharged proteins are significant harmfulness factors in plants and creatures. The parts of the sort II discharge framework are situated in both the inward and external layers where they amass into a multi-protein, cell-envelope traversing, complex. (28) The cell envelope of Gram-negative microscopic organisms is made out of two layers, which are isolated by the peptidoglycan-containing periplasm. While the envelope frames a basic obstruction against hurtful substances, it is by the by a compartment of extreme traffic for huge proteins, for example, compounds and poisons. Various examinations managing the sub-atomic component of protein discharge have uncovered that Gram-negative microbes advanced various techniques to accomplish this cycle. Among them, the sort II discharge system is essential for a twoventure measure. Exoproteins following this pathway are incorporated as sign peptide-containing antecedents. After cleavage of the sign peptide, the develop exoproteins are delivered into the periplasm, where they crease. The sort II hardware, otherwise called the secreton, is answerable for the movement of the periplasmic intermediates across the OM. The sort II framework is extensively preserved in Gram-negative microscopic organisms and includes a bunch of 12-16 unique proteins named GspC-M, GspAB, GspN, GspO, and GspS. The sort II discharge framework is exceptionally suggestive of the sort IV piliation gathering framework. In light of discoveries about the subcellular localisation of the Gsp parts, 
protein-protein associations among Gsps and their multimerisation status, underlying information and electron microscopy perception, it very well may be proposed a working model that strikingly runs the two frameworks in equal .(29) The sort III discharge framework (T3SS) is a macromolecular 'injectisome' that permits bacterial microbes to ship destructiveness proteins into the eukaryotic host cell. This macromolecular complex is made out of associated ring-like structures that length both bacterial films. The gem structures of the periplasmic space of the external layer secretin EscC and the internal film protein $\mathrm{PrgH}$ uncover the protection of a measured crease among the three proteins that structure the external film and inward layer rings of the T3SS.(30)

The sort III discharge framework (TTSS) of gram-negative microorganisms is answerable for conveying bacterial proteins, named effectors, from the bacterial cytosol straightforwardly into the inside of host cells. The TTSS is communicated dominatingly by pathogenic microorganisms and is generally used to bring malicious effectors into have cells. While biochemical exercises of effectors differ broadly, the TTSS mechanical assembly used to convey these effectors is saved and shows useful complementarity for emission and movement. A short area of mRNA succession or protein grouping in effectors goes about as a sign arrangement, coordinating proteins for transport through the TTSS. Moreover, various effectors require the activity of explicit TTSS chaperones for productive and physiologically significant movement into have cells. Various models clarifying how effectors are shipped into have cells have been proposed, yet comprehension of this cycle is fragmented and this point stays a functioning region of request. (31) Gram-negative microbes have an external film layer which compels take-up and discharge of solutes and polypeptides. To conquer this hindrance, microbes have built up a few frameworks for protein discharge. The sort $\mathrm{V}$ discharge pathway envelops the autotransporter proteins, the 
two-accomplice emission framework, and the as of late depicted sort Vc or AT-2 group of proteins. Nonetheless, the majority of the autotransporter proteins stay to be portrayed. Considering new disclosures and debates in this examination field, this audit considers the autotransporter emission measure with regards to the more broad field of bacterial protein movement and exoprotein work. (32)

The autotransporters, a group of discharged proteins from Gram-negative microbes, have a by and large bringing together structure including three utilitarian spaces: the amino-terminal pioneer succession, the emitted develop protein (traveler area) and a carboxy-terminal (beta-) space that frames a beta-barrel pore to permit emission of the traveler protein. Individuals from this family have been ensnared as significant or putative harmfulness factors in numerous Gram-negative microbes .(33) Bacterial adhesins intervene the connection of microorganisms to their specialties, for example, the tissue of a tainted host. Adhesins must be shipped across the phone envelope to get dynamic and during this discharge cycle they overlay into their last adaptation. This section centers around the biogenesis of the old style monomeric autotransporter proteins, which are the most pervasive class of discharged proteins in Gram-negative microorganisms. They may work as adhesins, yet different capacities are likewise known. Autotransporter proteins have a particular structure and comprise of a Nterminal sign peptide and a $\mathrm{C}$-terminal translocator space with in the middle of the emitted traveler area that harbors the capacities. The sign peptide coordinates the vehicle across the inward layer to the periplasm through the Sec hardware. The translocator area embeds into the external film and encourages the vehicle of the traveler to the cell surface. In this part, I will audit our present information on the discharge of traditional monomeric autotransporters and the strategies that have been utilized to survey their collapsing during the movement, both in vitro and in vivo. (34) 
4- Metabolic engineering of microbes initiate economic production of countless secondary metabolites which can be treated as the assets of mechanical raw materials and fuels. Plants are also critical targets to create essential auxiliary metabolites. Metabolic designing of both microorganism and plants too donate tall share in medicate discovery.so Nitty gritty information almost cell physiology, digestion system and/or metabolism is recommended In order to implement cumulative metabolic engineering techniques efficiently. (36) Extends the term of cell-free science, actualized both with decontaminated components and unrefined extricates, and is proceeding to extend our appreciation of organic premise whereas broadening the extend of applications. We are now not scared by the complexity of rough extricates and complicated response frameworks with hundreds of dynamic (36).

\subsection{Overview of cell-free metabolic engineering:}

This, in turn, luckily increments the extend of benefits displayed by unused items, both characteristic and extraordinary, that were already infeasible and/or unfathomable. But metabolic building by itself isn't adequate. Going forward, inventive metabolic building must be personally combinedwith inventive prepare building to completely realiz e potential commitments toward an economical worldwide civilization (37) Be that as it may, most victories of metabolic building have been kept to over-producing natively synthesized metabolites in E. coli and S. cerevisiae. A major reason for this advancement has been the plummet of metabolic building, especially auxiliary metabolic designing, to a collection of showings instead of a precise hone with generalizable devices. 
The development of the metabolites crossing natural layers is fundamentally controlled by the activity of the film potential. In biophysics, the intrigued is on the way in which the layer potential drives the molecule movement and makes more plausible its translocation, i.e., the travel through the channel and the elude through the inverse side with regard to the entrance. (38)

\subsection{Implementation of Metabolic engineering strategies:}

(38). The coordinate advancement of item arrangement and presenting of modern one through the alteration of particular biochemical responses considered as Metabolic designing definition but The foremost common implies of metabolic pathway building has been to target single rate-limiting steps which will control the surrender of conclusion items which Work at three levels: presenting biosynthetic qualities, utilizing translation variables and moving forward metabolic flux counting expanding the supply of strong, vitality, and diminishing control. (39). So by applying recent progress in biotechnology The coordinate advancement of item arrangement and presenting of modern one through the alteration of particular biochemical responses considered as Metabolic designing definition but The foremost common implies of metabolic pathway building has been to target single rate-limiting steps which will control the surrender of conclusion items which Work at three levels: presenting biosynthetic qualities, utilizing translation variables and moving forward metabolic flux counting expanding the supply of strong, vitality, and diminishing control .(38) reported that Directionality is a key to a successful metabolic engineering, but productivity is also important. Whereas plant generation frameworks are as a rule slower than microbial ones, plants are to a great extent autotrophic, and scale-up may thus be generally in costly, as production can use existing agricultural or horticultural infrastructure. However, for plant production 
systems to be effective, using agriculture or fermentation. Plants produce specialized compounds at certain times and in specific tissues. This means that usually their biosynthetic pathways are highly regulated and most regulation occurs at the transcriptional levels. Plant metabolic engineering can be implemented at different steps, the first is; new or modified biosynthetic genes are introduced, resulting in the generation of new or amended levels of target metabolites. The middle step is to enhance the transcription of genes encoding biosynthetic enzymes. (39) The last step of plant metabolic engineering is to improve the supply of substrates, energy, and reducing power.

\section{Conclusion:}

The use of secretion systems by bacteria to subvert host functions. Bacteria have evolved multiple systems to interact with and overcome their eukaryotic host and other prokaryotes. Secretion systems are required for the release of several effectors through the bacterial membrane(s) into the extracellular space or directly into the cytoplasm of the host, Where metabolic engineering has been defined as the direct improvement of product formation through the modification of specific biochemical reactions or the introduction of new ones, so it's implementation on microorganism and plant leading to drug discovery. Some critical steps enclose a well-organized and competent way and optimal metabolic engineering Moreover, the use genomics data for the activation of silent metabolic clusters can be incorporated.

\section{Abbreviations:}

TFs: transcription factors

\section{Declarations}




\section{Availability of data and material}

"Please contact author for data requests."

\section{Competing interests}

"The authors declare that they have no competing interests."

\section{Funding}

The authors have to funding to acknowledge.

\section{Acknowledgements}

The Authors Acknowledge the Spirit of Prof. Dr. Maisa, Mohamed Amin, the Nuclear Materials Authority as Our Home Institution,also Acknowledge National Organization for Drug Control and Research my family and, finally, my Favorite Drink (Coffee).

\section{Ethical approval and consent to participate}

Manuscript does not report studies involving human participants,

\section{Consent for publication}

Not Applicable

\section{Author's Contribution.}

Review has only one author. 


\section{Reference:}

1- Tomii K, Kanehisa M. A comparative analysis of $A B C$ transporters in complete microbial genomes. Genome Res. 1998 Oct;8(10):1048-59. doi: 10.1101/gr.8.10.1048. PMID: 9799792.

2- Davidson AL, Dassa E, Orelle C, Chen J. Structure, function, and evolution of bacterial ATP-binding cassette systems. Microbiol Mol Biol Rev. 2008 Jun;72(2):317-64, table of contents. doi: 10.1128/MMBR.00031-07. PMID: 18535149; PMCID: PMC2415747.

3- Ruiz N, Gronenberg LS, Kahne D, Silhavy TJ. Identification of two innermembrane proteins required for the transport of lipopolysaccharide to the outer membrane of Escherichia coli. Proc Natl Acad Sci U S A. 2008 Apr 
8;105(14):5537-42. doi: 10.1073/pnas.0801196105. Epub 2008 Mar 28.

PMID: 18375759; PMCID: PMC2291135.

4- Novikova M, Metlitskaya A, Datsenko K, Kazakov T, Kazakov A, Wanner B, Severinov K. The Escherichia coli Yej transporter is required for the uptake of translation inhibitor microcin C. J Bacteriol. 2007 Nov;189(22):8361-5. doi: 10.1128/JB.01028-07. Epub 2007 Sep 14. PMID: 17873039; PMCID: PMC2168686.

5- Tikhonova EB, Devroy VK, Lau SY, Zgurskaya HI. Reconstitution of the Escherichia coli macrolide transporter: the periplasmic membrane fusion protein MacA stimulates the ATPase activity of MacB. Mol Microbiol. 2007 Feb;63(3):895-910. doi: 10.1111/j.1365-2958.2006.05549.x. Epub 2007 Jan 4. PMID: 17214741.

6- Greene NP, Kaplan E, Crow A, Koronakis V. Antibiotic Resistance Mediated by the MacB ABC Transporter Family: A Structural and Functional Perspective. Front Microbiol. 2018 May 28;9:950. doi: 10.3389/fmicb.2018.00950. Erratum in: Front Microbiol. 2018 Sep 28;9:2318. PMID: 29892271; PMCID: PMC5985334.

7- Yamanaka H, Kobayashi H, Takahashi E, Okamoto K. MacAB is involved in the secretion of Escherichia coli heat-stable enterotoxin II. J Bacteriol. 2008 Dec;190(23):7693-8. doi: 10.1128/JB.00853-08. Epub 2008 Sep 19. PMID: 18805970; PMCID: PMC2583606.

8- Darbon E, Galinier A, Le Coq D, Deutscher J. Phosphotransfer functions mutated Bacillus subtilis HPr-like protein Crh carrying a histidine in the active site. J Mol Microbiol Biotechnol. 2001 Jul;3(3):439-44. PMID: 11361076.

9- Fujita Y. Carbon catabolite control of the metabolic network in Bacillus subtilis. Biosci Biotechnol Biochem. 2009 Feb;73(2):245-59. doi: 10.1271/bbb.80479. Epub 2009 Feb 7. PMID: 19202299.

10-Dahl U, Jaeger T, Nguyen BT, Sattler JM, Mayer C. Identification of a phosphotransferase system of Escherichia coli required for growth on $\mathrm{N}$ - 
acetylmuramic acid. J Bacteriol. 2004 Apr;186(8):2385-92. doi: 10.1128/jb.186.8.2385-2392.2004. PMID: 15060041; PMCID: PMC412175. 11- Morabbi Heravi K, Altenbuchner J. Cross Talk among Transporters of the Phosphoenolpyruvate-Dependent Phosphotransferase System in Bacillus subtilis. J Bacteriol. 2018 Sep 10;200(19):e00213-18. doi: 10.1128/JB.00213-18. PMID: 30038046; PMCID: PMC6148471.

12- Kotrba P, Inui M, Yukawa H. Bacterial phosphotransferase system (PTS) in carbohydrate uptake and control of carbon metabolism. J Biosci Bioeng. 2001;92(6):502-17. doi: 10.1263/jbb.92.502. PMID: 16233138.

13- Postma PW, Lengeler JW, Jacobson GR. Phosphoenolpyruvate:carbohydrate phosphotransferase systems of bacteria. Microbiol Rev. 1993 Sep;57(3):54394. PMID: 8246840; PMCID: PMC372926.

14-Gauthier A, Finlay BB. Bacterial pathogenesis: the answer to virulence is in the pore. Curr Biol. 2001 Apr 3;11(7):R264-7. doi: 10.1016/s0960-9822(01)00134-8. PMID: 11413015.

15-Kim JF. Revisiting the chlamydial type III protein secretion system: clues to the origin of type III protein secretion. Trends Genet. 2001 Feb;17(2):65-9. doi: 10.1016/s0168-9525(00)02175-2. PMID: 11173102.

16-Plano GV, Day JB, Ferracci F. Type III export: new uses for an old pathway. Mol Microbiol. 2001 Apr;40(2):284-93. doi: 10.1046/j.1365-2958.2001.02354.x. PMID: 11309112.

17-Rapisarda C, Fronzes R. Secretion Systems Used by Bacteria to Subvert Host Functions. Curr Issues Mol Biol. 2018;25:1-42. doi: 10.21775/cimb.025.001. Epub 2017 Sep 6. PMID: 28875938.

18-Burns DL. Type IV transporters of pathogenic bacteria. Curr Opin Microbiol. 2003 Feb;6(1):29-34. doi: 10.1016/s1369-5274(02)00006-1. PMID: 12615216.

19-Li YG, Hu B, Christie PJ. Biological and Structural Diversity of Type IV Secretion Systems. Microbiol Spectr. 2019 Mar;7(2):10.1128/microbiolspec.PSIB-0012- 
2018. doi: 10.1128/microbiolspec.PSIB-0012-2018. PMID: 30953428; PMCID: PMC6452883.

20-Alegria MC, Souza DP, Andrade MO, Docena C, Khater L, Ramos CH, da Silva AC, Farah CS. Identification of new protein-protein interactions involving the products of the chromosome- and plasmid-encoded type IV secretion loci of the phytopathogen Xanthomonas axonopodis pv. citri. J Bacteriol. 2005 Apr;187(7):2315-25. doi: 10.1128/JB.187.7.2315-2325.2005. PMID: 15774874; PMCID: PMC1065226.

21-Christie PJ, Vogel JP. Bacterial type IV secretion: conjugation systems adapted to deliver effector molecules to host cells. Trends Microbiol. 2000 Aug;8(8):354-60. doi: 10.1016/s0966-842x(00)01792-3. PMID: 10920394; PMCID: PMC4847720.

22-Christie PJ. Type IV secretion: intercellular transfer of macromolecules by systems ancestrally related to conjugation machines. Mol Microbiol. 2001 Apr;40(2):294-305. doi: 10.1046/j.1365-2958.2001.02302.x. PMID: 11309113; PMCID: PMC3922410.

23- Nunn D. Bacterial type II protein export and pilus biogenesis: more than just homologies? Trends Cell Biol. 1999 Oct;9(10):402-8. doi: 10.1016/s09628924(99)01634-7. PMID: 10481178.

24- Sandkvist M. Type II secretion and pathogenesis. Infect Immun. 2001 Jun;69(6):3523-35. doi: 10.1128/IAI.69.6.3523-3535.2001. PMID: 11349009; PMCID: PMC98326.

25- Sandkvist M. Biology of type II secretion. Mol Microbiol. 2001 Apr;40(2):27183. doi: 10.1046/j.1365-2958.2001.02403.x. PMID: 11309111.

26- Chernyatina AA, Low $\mathrm{HH}$. Core architecture of a bacterial type II secretion system. Nat Commun. 2019 Nov 28;10(1):5437. doi: 10.1038/s41467-01913301-3. PMID: 31780649; PMCID: PMC6882859. 
27- Delepelaire P. Type I secretion in gram-negative bacteria. Biochim Biophys Acta. 2004 Nov 11;1694(1-3):149-61. doi: 10.1016/j.bbamcr.2004.05.001. PMID: 15546664.

28- Hazes B, Frost L. Towards a systems biology approach to study type II/IV secretion systems. Biochim Biophys Acta. 2008 Sep;1778(9):1839-50. doi: 10.1016/j.bbamem.2008.03.011. Epub 2008 Mar 21. PMID: 18406342.

29- Johnson TL, Abendroth J, Hol WG, Sandkvist M. Type II secretion: from structure to function. FEMS Microbiol Lett. 2006 Feb;255(2):175-86. doi: 10.1111/j.1574-6968.2006.00102.x. PMID: 16448494.

30-Filloux A. The underlying mechanisms of type II protein secretion. Biochim Biophys Acta. 2004 Nov 11;1694(1-3):163-79. doi: 10.1016/j.bbamcr.2004.05.003. PMID: 15546665-[

31- Spreter T, Yip CK, Sanowar S, André I, Kimbrough TG, Vuckovic M, Pfuetzner RA, Deng W, Yu AC, Finlay BB, Baker D, Miller SI, Strynadka NC. A conserved structural motif mediates formation of the periplasmic rings in the type III secretion system. Nat Struct Mol Biol. 2009 May;16(5):468-76. doi: 10.1038/nsmb.1603. Epub 2009 Apr 26. PMID: 19396170; PMCID: PMC2860953.

32- Ghosh P. Process of protein transport by the type III secretion system. Microbiol Mol Biol Rev. 2004 Dec;68(4):771-95. doi: 10.1128/MMBR.68.4.771795.2004. PMID: 15590783; PMCID: PMC539011.

33- Henderson IR, Navarro-Garcia F, Desvaux M, Fernandez RC, Ala'Aldeen D. Type $\mathrm{V}$ protein secretion pathway: the autotransporter story. Microbiol Mol Biol Rev. 2004 Dec;68(4):692-744. doi: 10.1128/MMBR.68.4.692-744.2004. PMID: 15590781; PMCID: PMC539010.

34- Henderson IR, Navarro-Garcia F, Nataro JP. The great escape: structure and function of the autotransporter proteins. Trends Microbiol. 1998 Sep;6(9):3708. doi: 10.1016/s0966-842x(98)01318-3. PMID: 9778731. 
35- van Ulsen P. Protein folding in bacterial adhesion: secretion and folding of classical monomeric autotransporters. Adv Exp Med Biol. 2011;715:125-42. doi: 10.1007/978-94-007-0940-9_8. PMID: 21557061.

36- A. Dasgupta N. Chowdhury Rajat K. De 2020 Metabolic pathway engineering: Perspectives and applications Computer Methods and Programs in Biomedicine Volume 192, August 2020, 105436

37- James R. Swartz 2018 Expanding biological applications using cell-free metabolic engineering: An overview Metabolic Engineering Volume 50, November 2018, Pages 156-172 Author links open overlay panel ab

38-Rao Fu, Cathie Martin and Yang Zhang 2018 , Next-Generation Plant Metabolic Engineering, Inspired by an Ancient Chinese Irrigation System Molecular Plant 11, 47-57, January 2018.

39-M.J. Berridge, P. Lipp, M.D. Bootman, 2000. The versatility and universality of calcium signalling, Nat. Rev. Mol. Cell Biol. 1 (2000) 1121, http://dx.doi.org/ 10.1038/35036035. 